\title{
ON A CUBIC HECKE ALGEBRA ASSOCIATED WITH THE QUANTUM GROUP $U_{q}(2)$
}

\author{
JANUSZ WYSOCZAŃSKI \\ Institute of Mathematics, Wrocław University \\ Pl. Grunwaldzki 2/4, 50-384 Wroctaw, Poland \\ E-mail: jwys@math.uni.wroc.pl
}

\begin{abstract}
We define an operator $\alpha$ on $\mathbb{C}^{3} \otimes \mathbb{C}^{3}$ associated with the quantum group $U_{q}(2)$, which satisfies the Yang-Baxter equation and a cubic equation $\left(\alpha^{2}-1\right)\left(\alpha+q^{2}\right)=0$. This operator can be extended to a family of operators $h_{j}:=I_{j} \otimes \alpha \otimes I_{n-2-j}$ on $\left(\mathbb{C}^{3}\right)^{\otimes n}$ with $0 \leq j \leq n-2$. These operators generate the cubic Hecke algebra $\mathcal{H}_{q, n}(2)$ associated with the quantum group $U_{q}(2)$. The purpose of this note is to present the construction.
\end{abstract}

1. Introduction. In SLW3] Woronowicz considered the algebra of operators which intertwine the $n$-th tensor power of the fundamental representation $f_{N}$ of the quantum group $S U_{q}(N)$ with itself. For $n=2$ it is generated by

Here

$$
\sigma=I-\frac{\mu^{-2 N+4}}{[N-2]_{q^{2}} !}\left(I \otimes E^{*}\right)(E \otimes I) .
$$

$$
[N]_{q^{2}} !=\prod_{k=1}^{N} \frac{1-q^{2 k}}{1-q^{2}}
$$

and $E: \mathbb{C} \rightarrow\left(\mathbb{C}^{N}\right)^{\otimes N}$ and $E^{*}:\left(\mathbb{C}^{N}\right)^{\otimes N} \rightarrow \mathbb{C}$ are defined as

$$
E(1)=\sum_{k_{1}, \ldots, k_{N}=1}^{N} E_{k_{1}, \ldots, k_{N}} \cdot \varepsilon_{k_{1}} \otimes \ldots \otimes \varepsilon_{k_{N}}, \quad E^{*}\left(\varepsilon_{k_{1}} \otimes \ldots \otimes \varepsilon_{k_{N}}\right)=E_{k_{1}, \ldots, k_{N}}
$$

for the standard basis $\left\{\varepsilon_{1}, \ldots, \varepsilon_{N}\right\}$ of $\mathbb{C}^{N}$. This operator $\sigma$ can be written explicitly (see [SLW3, formula (4.13)) as

$$
\sigma\left(\varepsilon_{a} \otimes \varepsilon_{b}\right)= \begin{cases}q \cdot \varepsilon_{b} \otimes \varepsilon_{a} & \text { for } a<b \\ \varepsilon_{b} \otimes \varepsilon_{a} & \text { for } a=b \\ q \varepsilon_{b} \otimes \varepsilon_{a}+\left(1-q^{2}\right) \varepsilon_{a} \otimes \varepsilon_{b} & \text { for } a>b\end{cases}
$$

2000 Mathematics Subject Classification: 17B37, 20C08, 16T25.

Key words and phrases: quantum groups, cubic Hecke algebras, Yang-Baxter equation. The paper is in final form and no version of it will be published elsewhere. 
It has interesting properties, in particular it is a self-adjoint Hecke operator and satisfies the Yang-Baxter equation ([SLW3], (4.14)-(4.16)):

$$
\sigma^{*}=\sigma, \quad \sigma^{2}=\left(1-q^{2}\right) \sigma+q^{2} I, \quad(\sigma \otimes I)(I \otimes \sigma)(\sigma \otimes I)=(I \otimes \sigma)(\sigma \otimes I)(I \otimes \sigma)
$$

Therefore it defines a Hecke algebra $H_{q, n}$, generated by elements $\left\{g_{j}: j=1, \ldots, n\right\}$, if one puts

$$
g_{j}:=I_{j} \otimes \sigma \otimes I_{n-j-2} \quad \text { for } \quad j=1, \ldots, n-2,
$$

where $I_{k}$ denotes the identity map on $\left(\mathbb{C}^{N}\right)^{\otimes k}$. This Hecke algebra is exactly the algebra of intertwining operators for $f_{N}^{\otimes n}$.

2. The Yang-Baxter operator associated with $U_{q}(2)$. In this note we are going to show an analogous construction for the quantum group $U_{q}(2)$. The paper [W] contains a construction of the quantum group $U_{q}(2)$, in which the crucial role is played by the function counting the number of cycles in permutations from the symmetric group $S_{3}$. Namely, by considering the function $S_{3} \ni \sigma \mapsto(-q)^{3-c(\sigma)}$, where $c(\sigma)$ is the number of cycles and $q>0$, we constructed the following array:

$$
\begin{array}{ll}
E_{1,2,3}=1, & E_{1,3,2}=E_{2,1,3}=E_{3,2,1}=-q, \\
E_{2,3,1}=E_{3,1,2}=q^{2}, & E_{i, j, k}=0 \text { if }\{i, j, k,\} \varsubsetneqq\{1,2,3\} .
\end{array}
$$

This array defines an operator $\rho$ on $\mathbb{C}^{3} \otimes \mathbb{C}^{3}$ by

$$
\rho: \mathbb{C}^{3} \otimes \mathbb{C}^{3} \ni(a, b) \mapsto \sum_{i, j, k=1}^{3} E_{i, j, k} E_{k, a, b}(i, j) \in \mathbb{C}^{3} \otimes \mathbb{C}^{3}
$$

where $(a, b)$ denotes the standard basis element $\varepsilon_{a} \otimes \varepsilon_{b}$. In particular $\varepsilon_{1}=(1,0,0)$, $\varepsilon_{2}=(0,1,0)$ and $\varepsilon_{3}=(0,0,1)$.

The definition of $E$ implies that 2.4 simplifies to

$$
\rho(a, b)=E_{a, b, k} E_{k, a, b}(a, b)+E_{b, a, k} E_{k, a, b}(b, a), \quad \text { where } \quad\{a, b, k\}=\{1,2,3\}
$$

for $a \neq b$ and $a, b=1,2,3$. If $a=b$ then we get $\rho(a, a)=0$. The formulas can be written explicitly as follows.

$$
\begin{aligned}
& \rho(1,2)=E_{1,2,3} E_{3,1,2}(1,2)+E_{2,1,3} E_{3,1,2}(2,1)=q^{2}(1,2)+q^{3}(2,1), \\
& \rho(2,1)=E_{2,1,3} E_{3,2,1}(2,1)+E_{1,2,3} E_{3,2,1}(1,2)=q^{2}(2,1)+q(1,2), \\
& \rho(1,3)=E_{1,3,2} E_{2,1,3}(1,3)+E_{3,1,2} E_{2,1,3}(3,1)=q^{2}(1,3)+q^{3}(3,1), \\
& \rho(3,1)=E_{3,1,2} E_{2,3,1}(3,1)+E_{1,3,2} E_{2,3,1}(1,3)=q^{4}(3,1)+q^{3}(1,3), \\
& \rho(2,3)=E_{2,3,1} E_{1,2,3}(2,3)+E_{3,2,1} E_{1,2,3}(3,2)=q^{2}(2,3)+q(3,2), \\
& \rho(3,2)=E_{3,2,1} E_{1,3,2}(3,2)+E_{2,3,1} E_{1,3,2}(2,3)=q^{2}(3,2)+q^{3}(2,3) .
\end{aligned}
$$

Therefore, the operator $\alpha:=I_{2}-\frac{1}{q^{2}} \rho$ acts as: $\alpha(a, a)=(a, a)$ for $a=1,2,3$ and

$$
\begin{aligned}
& \alpha(1,2)=-q(2,1), \\
& \alpha(1,3)=-q(3,1), \\
& \alpha(3,2)=-q(2,3), \\
& \alpha(2,1)=-q^{-1}(1,2), \\
& \alpha(2,3)=-q^{-1}(3,2), \\
& \alpha(3,1)=\left(1-q^{2}\right)(3,1)-q(1,3) .
\end{aligned}
$$


This operator is not self-adjoint (which was the case for the Woronowicz's operator $\sigma-$ see 1.3 ), but $\alpha^{2}=\left(\alpha^{2}\right)^{*}$ is so, since

$$
\begin{aligned}
\alpha^{2}(1,2) & =(2,1) \\
\alpha^{2}(2,1) & =(2,1) \\
\alpha^{2}(2,3) & =(3,2) \\
\alpha^{2}(3,2) & =(2,3) \\
\alpha^{2}(1,3) & =q^{2}(1,3)-q\left(1-q^{2}\right)(3,1) \\
\alpha^{2}(3,1) & =\left(1-q^{2}+q^{4}\right)(3,1)-q\left(1-q^{2}\right)(1,3) .
\end{aligned}
$$

The first important property of $\alpha$ is that it is a Yang-Baxter operator.

Proposition 2.1. The operator $\alpha$ satisfies the Yang-Baxter equation

$$
(\alpha \otimes I)(I \otimes \alpha)(\alpha \otimes I)=(I \otimes \alpha)(\alpha \otimes I)(I \otimes \alpha) .
$$

Proof. Let $L=(\alpha \otimes I)(I \otimes \alpha)(\alpha \otimes I)$ be the left-hand side and $P=(I \otimes \alpha)(\alpha \otimes I)(I \otimes \alpha)$ be the right-hand side of $(2.8)$. We have to show that $L(a, b, c)=P(a, b, c)$ for every $a, b, c \in\{1,2,3\}$ (with the notation: $\left.(a, b, c)=\varepsilon_{a} \otimes \varepsilon_{b} \otimes \varepsilon_{c}\right)$. This requires checking 27 cases. It is clear that $L(a, a, a)=(a, a, a)=P(a, a, a)$ for any $a=1,2,3$. The direct calculation provides the following formulas for the other cases.

$$
\begin{aligned}
& L(3,2,3)=(3,2,3)=P(3,2,3), \\
& L(2,3,2)=(2,3,2)=P(2,3,2) \text {, } \\
& L(1,2,1)=(1,2,1)=P(1,2,1) \text {, } \\
& L(2,1,2)=(2,1,2)=P(2,1,2) \text {, } \\
& L(1,2,3)=-q(3,2,1)=P(1,2,3) \text {, } \\
& L(1,3,2)=-q^{3}(2,3,1)=P(1,3,2) \text {, } \\
& L(2,1,3)=-q^{-1}(3,1,2)=P(3,1,2) \text {, } \\
& L(3,3,2)=q^{2}(2,3,3)=P(3,3,2) \text {, } \\
& L(2,2,3)=q^{2}(3,2,2)=P(2,2,3) \text {, } \\
& L(3,2,2)=q^{2}(2,2,3)=P(3,2,2) \text {, } \\
& L(1,1,3)=q^{2}(3,1,1)=P(1,1,3) \text {, } \\
& L(1,3,3)=q^{2}(3,3,1)=P(1,3,3) \text {, } \\
& L(1,1,2)=q^{2}(2,1,1)=P(1,1,2) \text {, } \\
& L(1,2,2)=q^{2}(2,2,1)=P(1,2,2), \\
& L(2,3,3)=q^{-2}(3,3,2)=P(2,3,3), \\
& L(2,1,1)=q^{-2}(1,1,2)=P(2,1,1) \text {, } \\
& L(2,2,1)=q^{-2}(1,2,2)=P(2,2,1) \text {. } \\
& L(3,2,1)=\left(1-q^{2}\right)(3,2,1)-q(1,2,3)=P(3,2,1), \\
& L(3,1,2)=q^{2}\left(1-q^{2}\right)(2,3,1)-q^{3}(2,1,3)=P(3,1,2), \\
& L(2,3,1)=q^{-2}\left(1-q^{2}\right)(3,1,2)-q^{-1}(1,3,2)=P(2,3,1) \text {, } \\
& L(1,3,1)=-q\left(1-q^{2}\right)(3,1,1)+q^{2}(1,3,1)=P(1,3,1) \text {, } \\
& L(3,1,3)=-q\left(1-q^{2}\right)(3,3,1)+q^{2}(3,1,3)=P(3,1,3) \text {, }
\end{aligned}
$$




$$
\begin{aligned}
& L(3,1,1)=\left(1-q^{2}\right)(3,1,1)-q\left(1-q^{2}\right)(1,3,1)+q^{2}(1,1,3)=P(3,1,1), \\
& L(3,3,1)=\left(1-q^{2}\right)(3,3,1)-q\left(1-q^{2}\right)(3,1,3)+q^{2}(1,3,3)=P(3,3,1) .
\end{aligned}
$$

From these formulas the Proposition follows.

3. The cubic Hecke algebra associated with $U_{q}(2)$. The second important property of the operator $\alpha$ is that, even though it is not a Hecke operator, it does satisfy a cubic equation, and thus it generates a cubic Hecke algebra. This notion has been introduced by Funar in [F], where the cubic equation $\alpha^{3}-I=0$ was considered.

Proposition 3.1. The operator $\alpha$ satisfies the cubic equation

$$
\left(\alpha^{2}-I\right)\left(\alpha+q^{2} \cdot I\right)=0 .
$$

Proof. From the formulas 2.6, defining $\alpha$ it follows that it acts on the following subspaces by simple matricial formulas.

1. On the span of $(1,2),(2,1)$ as $\beta:=\left(\begin{array}{rr}0 & \frac{-1}{q} \\ -q & 0\end{array}\right)$.

2. On the span of $(2,3),(3,2)$ as $\beta^{*}:=\left(\begin{array}{rr}0 & -q \\ \frac{-1}{q} & 0\end{array}\right)$.

3. On the span of $(1,3),(3,1)$ as $\gamma:=\left(\begin{array}{rr}0 & -q \\ -q & 1-q^{2}\end{array}\right)$.

4. As identity on every $(a, a)$ with $a=1,2,3$.

It is straightforward to see that $\beta^{2}-I=0=\left(\beta^{*}\right)^{2}-I$. On the other hand, since

$$
\gamma^{2}=\left(\begin{array}{rr}
q^{2} & -q\left(1-q^{2}\right) \\
-q\left(1-q^{2}\right) & 1-q^{2}+q^{4}
\end{array}\right)
$$

we obtain

$$
\left(\gamma^{2}-I\right)\left(\gamma+q^{2} I\right)=\left(q^{2}-1\right)\left(\begin{array}{cc}
1 & q \\
q & q^{2}
\end{array}\right)\left(\begin{array}{cc}
q^{2} & -q \\
-q & 1
\end{array}\right)=\left(\begin{array}{ll}
0 & 0 \\
0 & 0
\end{array}\right)
$$

Therefore both $\beta$ and $\gamma$ satisfy the equation (3.12), so the $\alpha$ does.

Let us define the elements

$$
h_{j}:=I_{j} \otimes \alpha \otimes I_{n-j-2} \quad \text { for } \quad j=1, \ldots, n-2,
$$

where $I_{k}$ denotes the identity map on $\left(\mathbb{C}^{N}\right)^{\otimes k}$. Then by Propositions 2.1 and 3.1 the elements $h_{1}, \ldots, h_{n}$ generate a cubic Hecke algebra, associated with the quantum group $U_{q}(2)$.

Definition 3.2. The algebra $\mathcal{H}_{q, n}(2)$ generated by the elements $h_{j}, j=1,2, \ldots, n$ defined by (3.13) will be called the cubic Hecke algebra associated with the quantum group $U_{q}(2)$.

The basic properties of this algebra are summarized in the following. 
THEOREM 3.3. The generators $\left\{h_{j}: 1 \leq j \leq n\right\}$ of $H_{q, n}(2)$ satisfy:

$$
\begin{aligned}
h_{j} h_{j+1} h_{j} & =h_{j+1} h_{j} h_{j+1} & & \text { for } j=1, \ldots, n-1, \\
h_{j} h_{k} & =h_{k} h_{j} & & \text { for }|j-k| \geq 2, \\
\left(\left(h_{j}\right)^{2}-1\right)\left(h_{j}+q^{2}\right) & =0 & & \text { for } j=1, \ldots, n,
\end{aligned}
$$

The role of the Hecke algebra in the study of $S U_{q}(2)$ was that it was the intertwining algebra of the tensor powers of the fundamental co-representation. It is still to be checked whether the same role is played here by the $\mathcal{H}_{q, n}(2)$. In $[\mathrm{W}]$ the irreducible corepresentations have been described, but it was not clear if the description was complete. This problem will be studied elsewhere.

Acknowledgments. This research was partially supported by the European Commission Marie Curie Host Fellowship for the Transfer of Knowledge "Harmonic Analysis, Nonlinear Analysis and Probability" MTKD-CT-2004-013389, and the Polish Ministry of Science's research grants N N201364436 and N N201 270735.

\section{References}

[F] L. Funar, On the quotients of cubic Hecke algebras, Comm. Math. Phys. 173 (1995), 513-558.

[GR] G. Gasper and M. Rahman, Basic Hypergeometric Series, Encyclopedia of Mathematics and its Applications 35, Cambridge Univ. Press, 1990.

[JSW] P. E. T. Jørgensen, L. M. Schmitt and R. F. Werner, Positive representations of general commutation relations allowing Wick ordering, J. Funct. Anal. 134 (1995), 33-99.

$[\mathrm{PoW}]$ P. Podleś and S. L. Woronowicz, Quantum deformation of Lorentz group, Comm. Math. Phys. 130 (1990), 381-431.

[Ta] M. Takeuchi, A two-parameter quantization of $G L(n)$, Proc. Japan Acad. Ser. A Math. Sci 66 (1990), 112-114.

[SLW1] S. L. Woronowicz, Twisted SU(2) group. An example of non-commutative differential calculus, Publ. RIMS Kyoto Univ. 23 (1987), 117-181.

[SLW2] S. L. Woronowicz, Compact matrix pseudogroups, Comm. Math. Phys. 111 (1987), 613-665.

[SLW3] S. L. Woronowicz, Tannaka-Krein duality for compact matrix pseudogroups. Twisted SU(N) groups, Invent. Math. 93 (1988), 35-76.

[SLW4] S. L. Woronowicz, Compact quantum groups, in: Symétries quantiques (Les Houches, Session LXIV, 1995), North-Holland, Amsterdam, 1998, 845-884.

[W] J. Wysoczański, Twisted product structure and representation theory of the quantum group $U_{q}(2)$, Reports on Math. Phys. 54 (2004), 327-347. 
